This item was submitted to Loughborough's Research Repository by the author.

Items in Figshare are protected by copyright, with all rights reserved, unless otherwise indicated.

\title{
Fabrication and electrical properties of bulk textured LiCoO2
}

PLEASE CITE THE PUBLISHED VERSION

http://dx.doi.org/10.1111/j.1551-2916.2010.03634.x

\section{PUBLISHER}

(c) Wiley

\section{VERSION}

AM (Accepted Manuscript)

\section{PUBLISHER STATEMENT}

This work is made available according to the conditions of the Creative Commons Attribution-NonCommercialNoDerivatives 4.0 International (CC BY-NC-ND 4.0) licence. Full details of this licence are available at: https://creativecommons.org/licenses/by-nc-nd/4.0/

\section{LICENCE}

CC BY-NC-ND 4.0

\section{REPOSITORY RECORD}

Zhang, Hongtao, Peter J. Baker, and Patrick S. Grant. 2015. "Fabrication and Electrical Properties of Bulk Textured Licoo2". figshare. https://hdl.handle.net/2134/17744. 


\title{
Fabrication and electrical properties of bulk textured $\mathrm{LiCoO}_{2}$
}

\author{
Hongtao Zhang, ${ }^{1, *}$ Peter J. Baker ${ }^{2}$ and Patrick S. Grant ${ }^{1}$ \\ 1 Department of Materials, University of Oxford, Parks Road, Oxford OX1 3PH, UK \\ 2 Department of Physics, University of Oxford, Parks Road, Oxford OX1 3PU, UK
}

In order to study the orientation dependence of the electrical properties of layered structure cathode materials used in lithium-ion thin film microbatteries, a typical composition, $\mathrm{LiCoO}_{2}$, has been hot-press sintered in bulk with strong crystallographic texture. Microstructural characterization using X-ray diffraction, scanning electron microscopy and Raman spectroscopy showed that the degree of $\mathrm{LiCoO}_{2}$ texture depended upon the precise hot press conditions employed. There was a pronounced orientation of the polycrystalline $\mathrm{LiCoO}_{2} \mathrm{C}$-axis in the pressing direction with a high Lotgering factor $(f=0.96)$ for sintering at $950^{\circ} \mathrm{C}$. The pronounced texture led to a remarkable anisotropy in electronic conductivity between directions parallel $\left(\sigma_{/ /}\right)$and perpendicular $\left(\sigma_{\perp}\right)$ to the pressing direction in the temperature range 190-400 K, with $\sigma_{\perp} / \sigma_{/ /}=187$ at $300 \mathrm{~K}$. The results suggest that texture can be used to tune not only the well-known electrochemical performance of all-solid-state lithium-ion thin film batteries, but also their electronic conductivity.

\footnotetext{
Supported by the UK Engineering and Physical Sciences Research Council (EPSRC) for financial support under contract EP/D034256/1.

* Author to whom correspondence should be addressed. e-mail: hongtao.zhang@materials.ox.ac.uk
} 


\section{Introduction}

All-solid-state lithium-ion thin film microbatteries are used as micro-power sources in micro-sensors, micro-electronics and various miniaturised devices. ${ }^{1}$ There are two major groups of cathode materials for thin film microbatteries: layered structure and olivine structured compounds, which are exemplified by $\mathrm{LiCoO}_{2}$ and $\mathrm{LiFePO}_{4}$, respectively. ${ }^{2}$ Electrochemical and electrical properties of layered cathode materials will depend strongly on the orientation of the crystal due to their anisotropic crystallographic structure. For example, thin film $\mathrm{LiCoO}_{2}$ may exhibit a preferred crystallographic orientation dependent on the thickness of the film, and postdeposition annealing temperature and time. ${ }^{3-6}$ There have been investigations of the $\mathrm{LiCoO}_{2}$ film electrochemical behaviour with crystallographic orientation since this behaviour relates directly to battery performance. The orientations with (110) and (101) planes parallel to the substrate are the most favourable for a high Li intercalation/deintercalation rate. In these orientations, the Li planes are aligned parallel to the direction of current. ${ }^{3-6}$

However, in addition to sufficiently rapid Li-ion self-diffusion kinetics, another prerequisite property for high performance electrodes is a high electronic conductivity $(\sigma)$ in order to minimize internal cell resistance and to support high rate capability. ${ }^{2}$ Surprisingly, there are very few studies on the texture dependence of the electronic conductivity of layered cathode materials, which is of great significance when thin film microbatteries are considered. Takahashi et al. $^{7}$ reported an anisotropic temperature dependence of the electronic conductivity in single crystal $\mathrm{LiCoO}_{2}$ grown 
by a flux method, with conductivities measured perpendicular $\left(\sigma_{\mathrm{a}}\right)$ and parallel $\left(\sigma_{\mathrm{c}}\right)$ to the $c$-axis having an anisotropy of $\sigma_{\mathrm{a}} / \sigma_{\mathrm{c}} \approx 500$ at $300 \mathrm{~K}$. The temperature dependence of the in-plane conductivity $\left(\sigma_{\mathrm{a}}\right)$ suggested a variable range-hopping mechanism of conduction in the two-dimensional system. However in practice, thin film electrodes are composed of textured (preferred orientation) polycrystals. Systematic study of texture-electrical property relationships in these films is problematical because: (i) the films are usually made by physical vapour deposition, variable Li concentration often occurs $^{5,8}$ and even small deviations in stochiometry can produce large changes in electrical performance ${ }^{9}$ that partially or wholly obscure texture effects; and (ii) the texture cannot always be quantified by electron microscopy or X-ray diffraction based techniques because of low intensities and interference from the substrate. ${ }^{5}$ Therefore, we have developed an alternative processing route based on hot-press sintering that produces highly textured $\mathrm{LiCoO}_{2}$ in bulk so that multiple samples in different orientations can be taken from the same polycrystalline sample with no change in composition. The microstructures and the dependence of the bulk electronic conductivity on the induced texture and temperature is then investigated. We do not propose that hot-press sintering is a viable route to thin film batteries, but show that under carefully controlled and novel bulk processing conditions, textured polycrystalline $\mathrm{LiCoO}_{2}$ can be produced with control of chemistry and with remarkable electrical anisotropy of direct relevance to the understanding of thin film batteries.

\section{Experimental procedure}


Powdered $\mathrm{LiCoO}_{2}$ (Sigma-Aldrich Company Ltd) with stated purity of $99.8 \%$ was ball milled for $72 \mathrm{~h}$ and then sieved to under $250 \mu \mathrm{m}$. The powders were consolidated in a graphite die and sintered at a controlled temperature for $5 \mathrm{~h}$ under a uniaxial applied pressure of $20 \mathrm{MPa}$. Three sintering temperatures of $900 \pm 5^{\circ} \mathrm{C}, 950 \pm 5^{\circ} \mathrm{C}$ and $970 \pm 5^{\circ} \mathrm{C}$ were studied. The resulting discs were cut into thin bars with the long axis either parallel or perpendicular to the hot press direction. In order to remove any residual carbon picked up from the graphite die, the bars were annealed at $750^{\circ} \mathrm{C}$ for 10h in air. The relative density measured by Archimedes immersion was $96.9 \%$, $96.0 \%$ and $97.2 \%$ for the samples sintered at $900^{\circ} \mathrm{C}, 950^{\circ} \mathrm{C}$ and $970^{\circ} \mathrm{C}$ respectively. $\mathrm{X}$-ray diffraction $(\mathrm{XRD})$ using $\mathrm{Cu} K_{\alpha}$ radiation was performed at room temperature in a Siemens D5000 diffractometer. The microstructures of the ceramic samples were analyzed in a scanning electron microscope (SEM; JEOL 840A). The mole ratio of Li and Co was examined by inductively coupled plasma (ICP) emission spectroscopy (Perkin-Elmer OPTIMA 3000DV). Raman spectra were recorded using a Horiba Labram Aramis imaging confocal Raman microscope. Electronic conductivity was measured parallel $\left(\sigma_{/ /}\right)$and perpendicular $\left(\sigma_{\perp}\right)$ to the pressing direction in the temperature range 190-400 K using a Quantum Design Physical Property Measurement System (PPMS-14T). A four-probe arrangement was used with gold electrodes deposited by thermal evaporation on opposite end faces of the bar and point contact electrodes along the length of the bars.

\section{Results and discussion}

(1) Microstructural characterization of textured $\mathrm{LiCoO}_{2}$ 
XRD patterns from the $\mathrm{LiCoO}_{2}$ ceramic surfaces perpendicular to the pressing direction sintered at three different temperatures are shown in Fig. 1, and are compared with a pattern from the randomly oriented as-supplied powder. The diffraction peaks were matched and indexed according to the diffraction data of pure $\mathrm{LiCoO}_{2}$ (JCPDF No. 44-145). The materials were all single-phase within the sensitivity of the technique. For the powdered sample, the highest intensity reflection was (003) followed by (101) and (104) reflections [Fig. 1(a)], while for all the pressed $\mathrm{LiCoO}_{2}$ with the surface perpendicular to the pressing direction, the intensities of (003) reflection were much higher than all of other reflections indicating the development of texture along the $c$-axis of the $\mathrm{LiCoO}_{2}$ lattice. However, the three highest intensity reflections for each hot press temperature were different: (003), (006) and (104) for $900^{\circ} \mathrm{C}$ [Fig. 1(b)]; (003), (006) and (00 12) for $950^{\circ} \mathrm{C}$ [Fig. 1(c)]; and (003), (104) and (006) for $970^{\circ} \mathrm{C}$ [Fig. 1(e)]. The strongest texture developed at $950^{\circ} \mathrm{C}$ with a preferred orientation of the type $(00 l)$ where $l=3,6,9,12$, only. In comparison with the powder and $\mathrm{LiCoO}_{2}$ sintered at $950^{\circ} \mathrm{C}$ with the surface perpendicular to the pressing direction, $\mathrm{LiCoO}_{2}$ sintered at $950^{\circ} \mathrm{C}$ with the surface parallel to the pressing direction showed an increased intensity of (101) and (104) reflections, and a decreased (003) reflection intensity [Fig. 1(d)].

The degree of texture can be estimated using the Lotgering factor $f$ which was determined using the XRD data in the range $2 \theta=15^{\circ}-85^{\circ}$ according to: $:^{10}$

$$
f=\left(p-p_{0}\right) /\left(1-p_{0}\right)
$$

where $p=\sum_{l} I(00 l) / \sum_{h k l} I(h k l), \sum_{l} I(00 l)$ and $\sum_{h k l} I(h k l)$ were the sums of the intensities of $(00 l)$ and $(h k l)$ reflections respectively, and $p_{0}$ is the value of $p$ for a 
randomly oriented sample, taken in this study as the as-supplied powder. The $f$ values for $\mathrm{LiCoO}_{2}$ sintered at $900^{\circ} \mathrm{C}, 950^{\circ} \mathrm{C}$ and $970^{\circ} \mathrm{C}$ were $0.61,0.96$, and 0.51 respectively.

Fig. 2 shows SEM images of fractured cross-section surfaces parallel to the pressing direction for all the sintering temperatures. There are dramatic differences in terms of the morphology and alignment of grains. As shown in Fig. 2(a), the microstructure sintered at $900^{\circ} \mathrm{C}$ exhibited a mixture of plate-like grains aligned to the pressing direction and equiaxed grains with dimensions of $1 \sim 3 \mu \mathrm{m}$. In contrast, $\mathrm{LiCoO}_{2}$ sintered at $950^{\circ} \mathrm{C}$ displayed almost all plate-like grains in which the short axis was aligned strongly to the pressing direction [Fig. 2(b)]. Most of the equiaxed grains were consumed during the growth of these plate-like grains. The average plate-like $\mathrm{LiCoO}_{2}$ grain size sintered at $950^{\circ} \mathrm{C}(15 \mu \mathrm{m}$ length and $4 \mu \mathrm{m}$ thickness $)$ was much larger than that sintered at $900^{\circ} \mathrm{C}(7 \mu \mathrm{m}$ length and $1.5 \mu \mathrm{m}$ thickness $)$. Aligned platelike $\mathrm{LiCoO}_{2}$ grains remained when sintered at $970^{\circ} \mathrm{C}$, as shown in Fig. 2(c). However, when compared with $950^{\circ} \mathrm{C}$, the anisotropy in grain aspect ratio decreased at $970^{\circ} \mathrm{C}$ because of the development of coarser more equiaxed grains. Overall, the variation in the Lotgering factor $f$ could be readily correlated to the development of elongated grains of increasing aspect ratio, with the highest value of $f$ at $950^{\circ} \mathrm{C}$ corresponding to well-aligned and uniform plate-like grains. There was no systematic variation in micro-porosity in a particular direction and no measureable change in density for the three temperatures presented here.

The presence of the $\mathrm{Co}_{3} \mathrm{O}_{4}$ in $\mathrm{LiCoO}_{2}$ is often encountered because of evaporation loss of $\mathrm{Li}$ during elevated temperature processing. ${ }^{5,8} \mathrm{Co}_{3} \mathrm{O}_{4}$ has high electronic conductivity $^{11}$ that could affect subsequent electrical measurements. The $\mathrm{Li} / \mathrm{Co}$ atomic 
ratio obtained by ICP for $\mathrm{LiCoO}_{2}$ sintered at $950^{\circ} \mathrm{C}$ was 0.98 and close to stoichiometry. Raman spectroscopy was also used to examine further the $950^{\circ} \mathrm{C}$ hotpressed texture and possible impurity effects since it is more sensitive to minority phases that may be below the detection limit in XRD. Fig. 3 shows Raman spectra from the polished surface of textured $\mathrm{LiCoO}_{2}$ sintered at $950^{\circ} \mathrm{C}$ both perpendicular and parallel to the pressing direction. The spectra showed two Raman active modes at 488 and $597 \mathrm{~cm}^{-1}$ assigned as $\mathrm{E}_{\mathrm{g}}$ and $\mathrm{A}_{1 \mathrm{~g}}$ respectively, which characterized layered $\mathrm{LiCoO}_{2}{ }^{8}$ The absence of $\mathrm{Co}_{3} \mathrm{O}_{4}$ impurity phase was evidenced by the absence of any peak at $690 \mathrm{~cm}^{-1}$. The intensity ratio $\mathrm{I}_{597} / \mathrm{I}_{488}$ increased with the degree of $c$-axis orientation: 0.72 for the surface parallel to the pressing direction and 1.58 for the surface perpendicular to the pressing direction, and in agreement with similar measurements of $\mathrm{LiCoO}_{2}$ thin films. ${ }^{5}$

(2) The effect of texture on the electronic conductivity of polycrystalline $\mathrm{LiCoO}_{2}$

Fig. 4 shows the temperature dependence of the electronic conductivity of the $\mathrm{LiCoO}_{2}$ bars sintered at $950^{\circ} \mathrm{C}$. The strong dependence with temperature evidenced thermally activated semi-conducting behaviour in both orientations, and there was strong, nearly temperature-independent electronic conductivity anisotropy. The conductivity in the temperature range 190-400 K did not strictly obey often-assumed Arrhenius-type behaviour, as shown in the inset of Fig. 4. The $\mathrm{LiCoO}_{2}$ electronic conductivities at $300 \mathrm{~K}$ were $1.68 \times 10^{-3} \mathrm{~S} / \mathrm{cm}$ for $\sigma_{\perp}$ and $8.97 \times 10^{-6} \mathrm{~S} / \mathrm{cm}$ for $\sigma_{/ /}$. The anisotropy of conductivity $\sigma_{\perp} / \sigma_{/ /}$was 187 at $300 \mathrm{~K}$ and clearly indicated that the main conduction path was along the $a-b$ plane, as previously reported for single crystal $\mathrm{LiCoO}_{2}{ }^{7}$ 
However, similar behaviour has not been reported previously for textured polycrystalline $\mathrm{LiCoO}_{2}$.

$\mathrm{LiCoO}_{2}$ conductivity is provided by the variable-range hopping (VRH) mechanism arising from the localized Co-3d electrons in the valance band. ${ }^{12}$ According to the VRH theory of conduction:

$$
\sigma \propto \exp \left[-\left(T_{0} / T\right)\right]^{1 /(d+1)}
$$

where $d$ is the dimension of the conduction path and $T_{0}$ is the Mott characteristic temperature. ${ }^{13}$ The $\sigma_{\perp}$ data from the $a-b$ plane were best-fitted to Equation (2) using a least-squares regression that yielded $d=2.94$ with a regression coefficient $R^{2}=$ 0.99993. For comparison, if it was assumed $d=3$ or $d=2$, then $R^{2}=0.99991$ and 0.98471, respectively. Fig. 4 shows the close fit to Equation (2) for $d=3$. Given the very high degree of texture, it is at first slightly surprising that conduction in the highly aligned $a-b$ plane should be suggested to be 3-dimensional, particularly since the $a-b$ plane electronic conductivity in single crystal $\mathrm{LiCoO}_{2}$ is reported as 2dimensional with $d=2 .^{7}$ An immediate explanation for the different dimensionality of $a-b$ plane conduction in single $(d=2)$ and polycrystalline $(d=3) \mathrm{LiCoO}_{2}$ might implicate the role of grain boundaries, or the non-perfect alignment of the $a-b$ plane in the textured polycrystalline material. However, there are at least two other reports of $d$ $=3$ for single crystal $\mathrm{CuAlO}_{2}{ }^{14}$ and $\mathrm{LiVO}_{2}{ }^{15}$ both of which have a similar layered structure and belong to the same space group as $\mathrm{LiCoO}_{2}$. Therefore, grain boundaries alone may not be the only important consideration in explaining anisotropic conduction behaviour in polycrystalline materials of this type. Although the XRD and Raman data in Figures 1 and 3 suggested there was close control of stochiometry, we 
tentatively suggest that even very minor changes in composition may have a role in the apparent mechanism of electron conduction.

Although the present study focused on bulk $\mathrm{LiCoO}_{2}$ rather than thin films, it is reasonable to expect similar anisotropic electronic conductivity in textured cathode thin films with layered structure. As stated before, studies of $\mathrm{LiCoO}_{2}$ films preferred orientations and electrochemical behaviour showed that the (110) and (101) orientations are most favourable for a high $\mathrm{Li}$ intercalation/deintercalation rate, whereas the (003) orientation is unfavourable. ${ }^{3}$ The present study suggests that from the viewpoint of previously unstudied electrical properties, polycrystalline layered structure cathode thin films with orientations further away from the $c$-axis (or close to the $a$-b-axis), offer the best performance for thin film batteries because they exhibit much higher electronic conductivity. A key deduction from our results is that polycrystalline layered structure cathode thin films with orientations close to the $a-b-$ axis are highly desirable not only from the viewpoint of the associated well-known improvement in electrochemical behaviour, but also from that of electronic conductivity with particular ramifications in thin film microbatteries.

\section{Conclusions}

An anisotropic electronic conductivity in polycrystalline cathode materials of lithium ion batteries with layered structure has been demonstrated through one typical compound, $\mathrm{LiCoO}_{2}$. Bulk polycrystalline $\mathrm{LiCoO}_{2}$ has been fabricated with deliberately induced crystallographic texture by hot-press sintering. $\mathrm{LiCoO}_{2}$ sintered at $950^{\circ} \mathrm{C}$ has a plate-like microstructure with a strong orientation of the $c$-axis in the 
pressing direction and a high Lotgering factor of $f=0.96$, with a two-order of magnitude anisotropy in the electronic conductivity $\left(\sigma_{\perp} / \sigma_{/ /}\right)$and the main conduction path within the $a-b$ plane in the range $190-400 \mathrm{~K}$. Our results suggest that texture can be used to tune not only the well-known electrochemical performance of all-solidstate lithium-ion thin film batteries, but also their electrical properties.

\section{References}

${ }^{1}$ J. B. Bates, N. J. Dudney, B. Neudecker, A. Ueda, and C. D. Evans, "Thin-Film Lithium and Lithium-Ion Batteries," Solid State Ionics., 135 [1-4] 33-45 (2000).

${ }^{2}$ M. S. Whittingham, "Lithium Batteries and Cathode Materials," Chem. Rev., 104 [10] 4271-302 (2004).

3 J. B. Bates, N. J. Dudney, B. J. Neudecker, F. X. Hart, H. P. Jun, S. A. Hackney, "Preferred Orientation of Polycrystalline $\mathrm{LiCoO}_{2}$ Films,” J. Electrochem. Soc., 147 [1] 59-70 (2000).

${ }^{4}$ J. Xie, N. Imanishi, T. Matsumura, A. Hirano, Y. Takeda, O. Yamamoto, “Orientation Dependence of Li-ion Diffusion Kinetics in $\mathrm{LiCoO}_{2}$ Thin Films Prepared by RF Magnetron Sputtering," Solid State Ionics., 179 [9-10] 362-70 (2008).

${ }^{5}$ Y. Iriyama, M. Inaba, T. Abe, Z. Ogumi, "Preparation of $c$-axis Oriented Thin Films of $\mathrm{LiCoO}_{2}$ by Pulsed Laser Deposition and Their Electrochemical Properties," J. Power Sources., 94 [2] 175-82 (2001).

${ }^{6}$ P. J. Bouwman, B. A. Boukamp, H. J. M. Bouwmeester and P. H. L. Notten, "Structure-Related Intercalation Behaviour of $\mathrm{LiCoO}_{2}$ Films," Solid State Ionics., 152-153 181-88 (2002).

7 Y. Takahashi, Y. Gotoh, J. Akimoto, S. Mizuta, K. Tokiwa, and T. Watanabe, “Anisotropic Electrical Conductivity in $\mathrm{LiCoO}_{2}$ Single Crystal," J. Solid State Chem., 164 [1] 1-4 (2002).

${ }^{8}$ M. Inaba, Y. Iriyama, Z. Ogumi, Y. Todzuka and A. Tasaka, "Raman Layered Rock-Salt $\mathrm{LiCoO}_{2}$ and its Electrochemical Lithium Deintercalation,” J. Raman Spectrosc., 28 [8] 613-17 (1997).

9 M. Ménétrier, I. Saadoune, S. Levasseur, C. Delmas, “The Insulator-Metal Transition upon Lithium Deintercalation from $\mathrm{LiCoO}_{2}$ : Electronic Properties and ${ }^{7} \mathrm{Li}$ NMR Study," J. Mater. Chem., 9 [5] 1135-40 (1999). 
${ }^{10}$ T. Takenaka and K. Sakata, "Grain Orientation and Electrical Properties of Hot-Forged $\mathrm{Bi}_{4} \mathrm{Ti}_{3} \mathrm{O}_{12}$ Ceramics,” Jpn. J. Appl. Phys., 19 [1] 31-9 (1980).

${ }^{11}$ T. Tennakoon, G. Lindbergh, B. Bergman, "Performance of $\mathrm{LiCoO}_{2}$ Cathode, Prepared Using the Pechini Method, in Molten Carbonate Fuel Cell,” J. Electrochem. Soc. 144 [7] 2296-301 (1997).

${ }^{12}$ K. Kushida and K. Kuriyama, "Mott-Type Hopping Conduction in the Ordered and Disordered Phases of $\mathrm{LiCoO}_{2}$," Solid State Commun., 129 [8] 525-28 (2004).

${ }^{13}$ B. Kramer and A. MacKinnon, "Localization: Theory and Experiment," Rep. Prog. Phys., 56 [12] 1469-564 (1993).

${ }^{14}$ M. S. Lee, T. Y. Kim, and D. Kim, “Anisotropic Electrical Conductivity of Delafossite-Type $\mathrm{CuAlO}_{2}$ Laminar Crystal,” Appl. Phys. Lett., 79 [13] 2028-30 (2001).

${ }^{15}$ H. Takei, M. Koike, K. Imai, H. Sawa, H. Kadowaki and Y. Iye, "Growth and Properties of LiDeficient Lithium Vanadium Dioxide Single Crystal," Mater. Res. Bull., 27 [5] 555-62 (1992). 


\section{Figure captions}

Fig. 1. XRD patterns of $\mathrm{LiCoO}_{2}$ : (a) as-supplied powder; and hot pressed $\mathrm{LiCoO}_{2}$ sintered at (b) $900^{\circ} \mathrm{C}$ with the surface perpendicular to the pressing direction; (c) $950^{\circ} \mathrm{C}$ with the surface perpendicular to the pressing direction; (d) $950^{\circ} \mathrm{C}$ with the surface parallel to the pressing direction; and (e) $970^{\circ} \mathrm{C}$ with the surface perpendicular to the pressing direction.

Fig. 2. SEM micrographs from the fractured cross-section surfaces of $\mathrm{LiCoO}_{2}$ parallel to the pressing direction surface: sintered at (a) $900^{\circ} \mathrm{C}$, (b) $950^{\circ} \mathrm{C}$, and (c) $970^{\circ} \mathrm{C}$.

Fig. 3. Raman spectra of textured $\mathrm{LiCoO}_{2}$ sintered at $950^{\circ} \mathrm{C}$ with the polished surface (a) perpendicular and (b) parallel to the pressing direction.

Fig. 4. $\sigma$ vs. $T^{1 / 4}$ plots for the textured $\mathrm{LiCoO}_{2}$ sintered at $950^{\circ} \mathrm{C}$ and measured parallel $\left(\sigma_{/ /}\right)$and perpendicular $\left(\sigma_{\perp}\right)$ to the pressing direction. Inset is on Arrhenius plots of $\sigma \mathrm{vs} .1000 / \mathrm{T}$. 


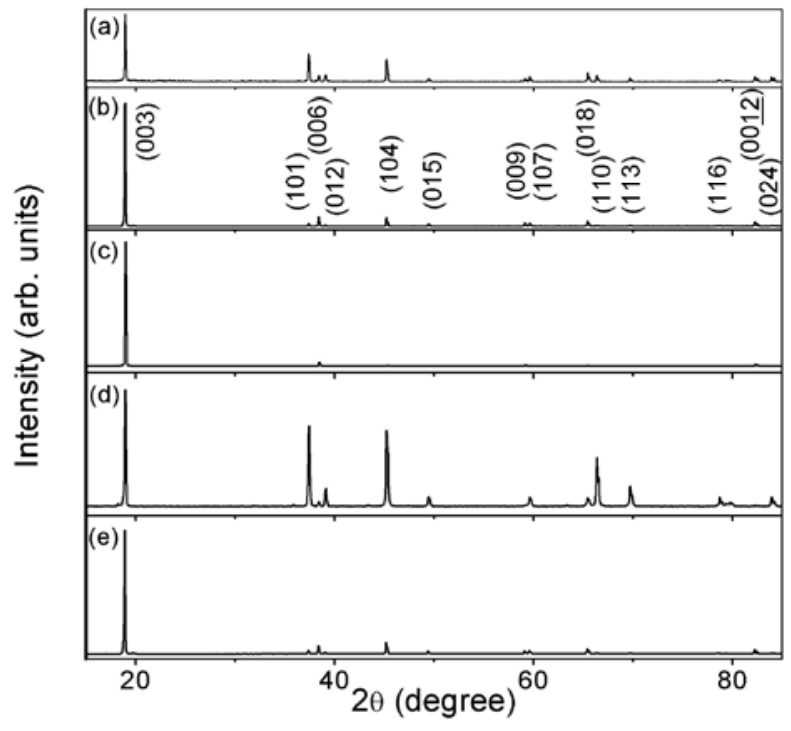

Fig. 1
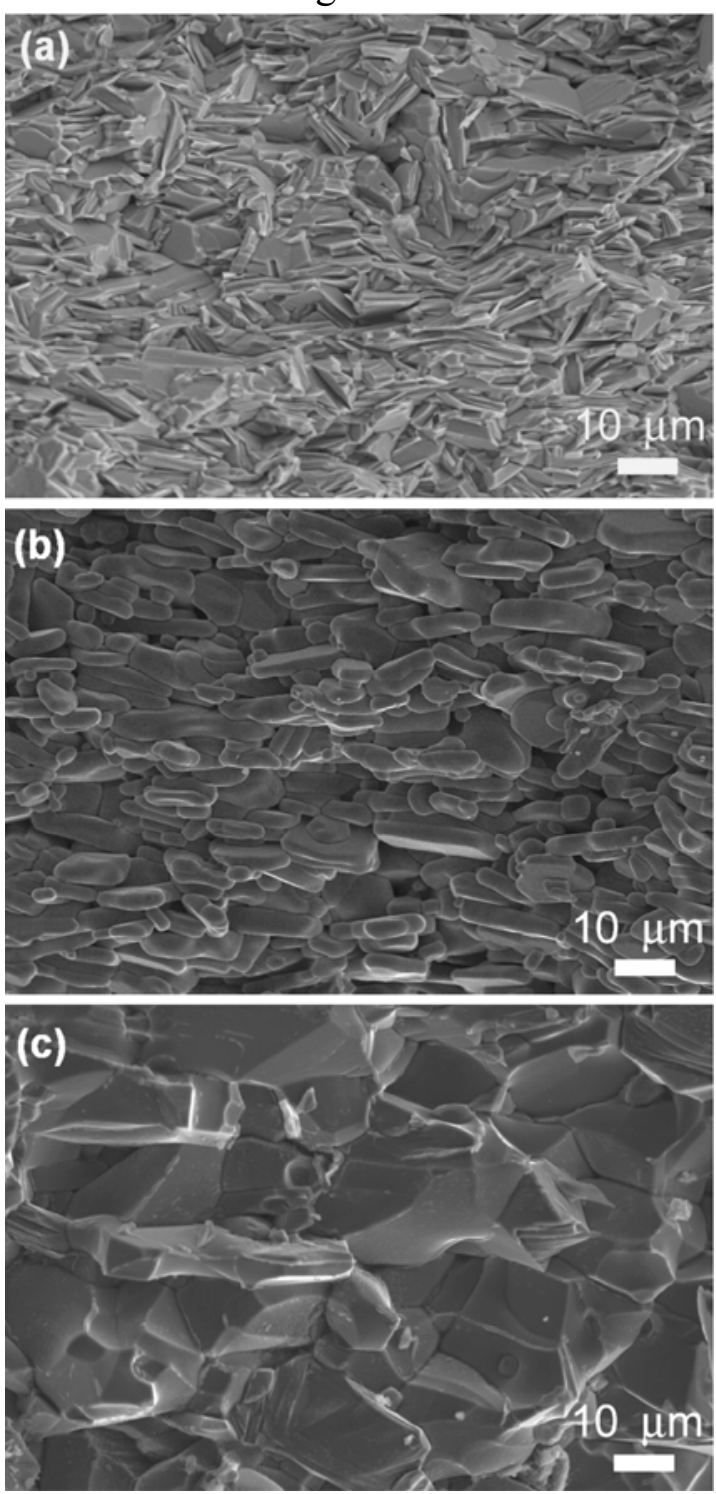

Fig. 2 


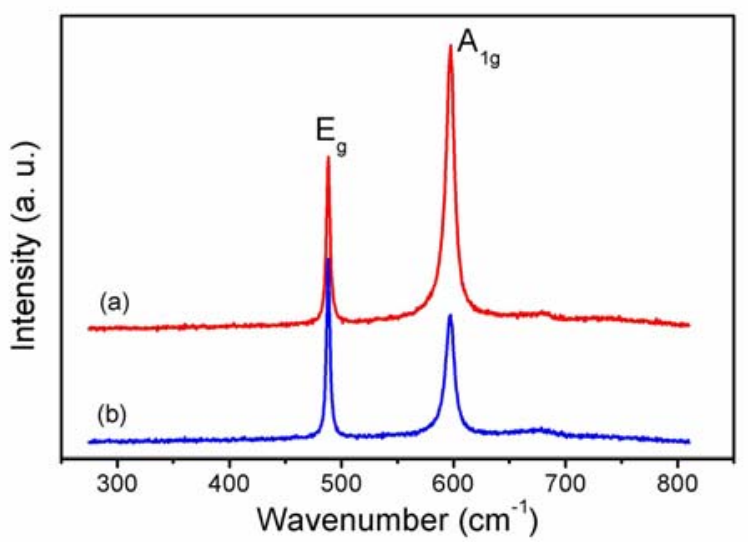

Fig. 3

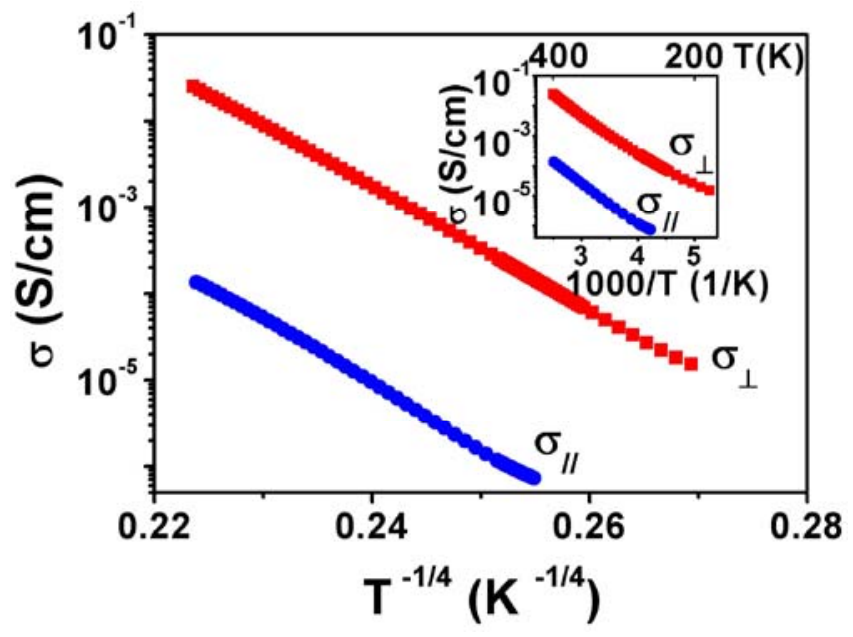

Fig. 4 